\title{
New Trend in Disintegration Process of Clay in Placer
}

\author{
Alexander G Mikhailov* \\ Mineral Resources Laboratory of the Institute of Chemistry and Chemical Technology of Siberian Branch of the Russian Academy of Sciences, Russia \\ *Corresponding author: Alexander G Mikhailov, Mineral Resources Laboratory of the Institute of Chemistry and Chemical Technology of Siberian \\ Branch of the Russian Academy of Sciences, Krasnoyarsk, Krasnoyarsk region, Russia
}

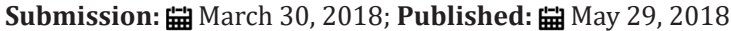

\begin{abstract}
The power estimation of a disintegration process of clay is given based on the physical phenomena of interaction of thin particles in the clay unit The method of volumetric processing of the disintegration process is proposed. Now the perspective directions in ore dressing are the acoustic method. An acoustic method of clay processing allows treatment all volumes of materials. The results of experimental researches of various types of clay raw material are given. The power parameters of a disintegration process are estimated.
\end{abstract}

Keywords: Clay; Disintegration; Particle binding structure

Highlights

a. The acoustic principle of disintegration is due to the proportionality of the particle size, the nature of the connection between particles in clay aggregates and wave low-frequency processing;

b. Complete disintegration of clay is possible with the use of three-dimensional processing;

c. The energy costs of real disintegration are 2 to 3.5 times higher than the theoretical

\section{Introduction}

The receipt of commercial products from the mineral resources of clay materials has a wide range of applications: gold from placers, the production of ceramic products, refractory materials, building materials, etc. When working with clay, the first operation is disintegration. Applied disintegration equipment in technological complexes has high energy indices [1-4]. Virtually all technological processes are based on the mechanical principle of processing. The existing methods of clay disintegration are very energy-consuming and labor-intensive. Moreover, the equipment for disintegration has a restriction on the content of clay. Thus, technological complexes for extracting gold from placers cannot be profitable for the development of sands with a clay content of more than $25-40 \%$ due to high losses with incomplete disintegration. To date, almost all available placers with a clay content of less than $50 \%$ have been worked out.

However, significant gold reserves remain in the primary zones of weathering (oxidation zone) and in placer deposits with high clay content [5]. A characteristic feature of the weathering crust is high clay content (up to 90-100\%). The gold content in weathering crusts can be $5 \mathrm{~g} / \mathrm{t}$ and more [6]. The involvement of such deposits in operation is possible with a reduction in costs for the process of disintegration. If we take into account the nature of particle bonds in clay aggregates, then we must abandon the mechanical principle of destruction of clay aggregates. For such small sizes of clay particles $(1-3 \mu \mathrm{m})$ disintegration based on mechanical treatment of the material will not ensure complete disintegration. The clay material is $[7,8]$ a set of mineral particles physically bound in a common skeleton through water. Water in the structure of clay plays the main binding role. Water can be in the adsorbed state and under the conditions of film water (Figure 1).

In a layer of adsorbed water, allocate a layer snug against the surface of a particle, which have properties like a solid body (thickness 1-3 molecules) and somatic layer having some mobility (thickness 10-20 molecules). The film water forms a film atop adsorbed water. The film water presence is determined by the excess of humidity of the breed above by its maximal hygroscopicity. A more rational methods, which are commensurate with the particle size in clay aggregates, for disintegration are methods of volume effect [7-9]. To assess the applicability of the methods of volumetric disintegration, it is necessary to estimate the magnitude of the energy impact for breaking the bonds between the particles. 


\section{Estimation of power consumption at disintegration process}

The mineral particles of aggregate are strongly kept in a skeleton by intermolecular forces of an attraction. Basically, it is forces of a superficial tension. In clay material than the particle has less in size, then the more it has of relative surfaces. Consequently, it has stronger contact to adjacent particles. The clay aggregate strength provides the contact of particles among themselves through an area of a layer adsorbed water. The connection of particles through the medium of film water will cause their relative mobility. The connection of clay particles through the medium of free water will cause their full independence in the system. The connection forces are determined by the power of one contract and their number, and do not depend from properties of clay particles in the mix.

The mathematical expression 9 determines the force of one contact between clay particles of the spherical form:

$$
F=A \cdot a / 12 \cdot m^{2}
$$

Where: A-Van der Waals' constant (takes into account a nature of cooperating bodies), a-radius of an element, m-thickness of dispersion layer of medium between particles. The potential energy of an attraction between two particles of the spherical form is determined by the expression:

$$
q=-A \cdot a / 12 m
$$

The specific potential energy of interaction will be determined by the following expression:

$$
Q_{o}=-\chi_{o} \frac{A \cdot a}{12 \cdot m}
$$

Where: $\chi_{0}$ - common number of contacts of interaction.

The clay particles have the various forms and sizes [8]. The greatest connection force of particles is observed in their interaction at a level of enclosure-adsorbed water (aggregate condition of clay). Kinetic energy of on the level of adsorbed water corresponds to the quantity of the heat of wetting a surface

$\left(116 \cdot 10^{-3} \mathrm{~J} / \mathrm{m}^{2}\right)$ (Figure 1). The least force interaction lies in the limits of a double layer of film water (Figure 1). The full absence of connection between particles may be achieved if a presence of adjacent particles is outside distance of a double layer of adsorbed water plus double layer of film water (Figure 1) (absolute disintegration).

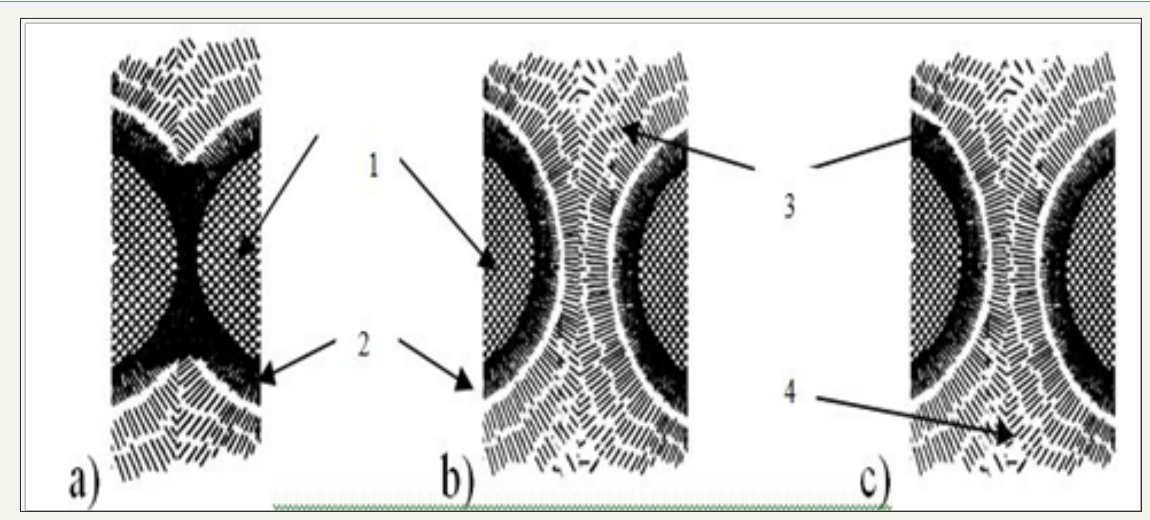

1-Particle; 2-Adsorbed water; 3-Film water; 4-Free water

Figure 1: The circuit of probable connections of particles clay in water medium.

Absolute disintegration of clay requires power consumption in size of destruction energy of all connections of interaction. The volume of disintegration energy will have an essential difference depending on a share of clay aggregates presence of units in an initial material and from the sizes of initial clay particles. The absolute disintegration of clay aggregates of kaolin clay from the account of destruction of Van der Waals' attractive force will occur through applying of energy of the order $\sim 300 \mathrm{~kJ} / \mathrm{m}^{3}$.

The disintegration essence consists in achievement of complete disclosing of surface of extracting minerals. For the technological purposes the achievement absolute clay disintegration has not always been necessary. For example, the washing of a lump material, should supply removal clay pug from a surface of the pieces. Thus, the part of a clay material can remain in a clay aggregate condition and it is not reflected in result of washing. At gravitational extraction of gold from gold-bearing sand the disintegration is necessary for all material up to the minimal size in $\sim 30$ micron. For gold extraction by flotation method the size of particles in pulp should not exceed 3-5 micron. The basic result of a process of washing is the achievement of full disintegration for further technological operation with mineral raw material. Thus, a degree of disintegration, which suffice of complete surface disclosing of extracting component for used technology of processing has name as full disintegration.

The necessary values of energy for full disintegration will be essential to change depending on required product size. The rough characteristic of energy expenses for disintegration of kaolin clay depending on the various sizes of particle is given in (Figure 2). The greatest energy $\left(\sim 400 \mathrm{~kJ} / \mathrm{m}^{3}\right)$ is required to be applied for clay disintegration up to product size in 1 micron. Such order of size of power expenses is characteristic for aggregating (adsorbed) of a clay condition trend 1). At initial loosely packed clay condition the 
disintegration energy up to the similar sizes, is reduced essentially $\left(\sim 70 \mathrm{KJ} / \mathrm{m}^{3}\right)$ (Figure 2), (trend 2). The results of experimental data of full destruction of connections between clay particles in bentonite clay by shock wave method [7], researchers of acoustic technology in field of dressing [9] and our experiments confirm the order of size power consumption of clay disintegration.

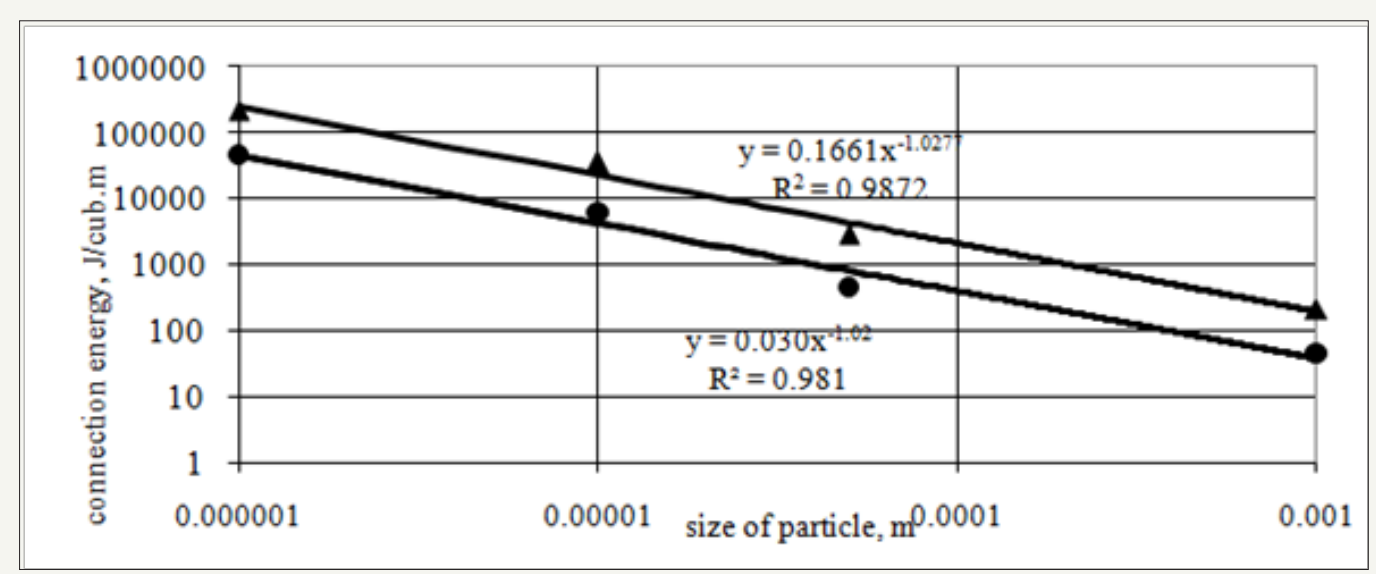

Figure 2: Depending on the energy volume for full disintegration for the size.

The characteristic of power consumption for the clay disintegration process is based on theoretical account of the forces of a superficial tension of Van der Waals'. Thus, the estimation of meanings of power disintegration consumption represents a theoretical account of the ideal process of unitary destruction of influence contact forces. Given the power characteristics of a disintegration process, characterize the order of size of energy, which is necessary for applying for loosening of particles.

The real disintegration process represents the more complex mechanism. First, the all clay materials have thyrotrophic properties. The restoration of the broken connections between particles takes place always and very quickly. The speed of their renewal after infringement is commensurable with the speed of the break. The regeneration of connections between particles can occur through the covering of adsorbed and film water and to become isolated only on a layer of free water. Only at connections on free water there is no mutual influence of particle. The achieved condition of relative freedom of particles is possible during the long period of time. As a rule, it is enough of this period of time not only for the subsequent technological process of extraction, but also it is required of additional technical and technological intervention for directed sedimentation in slurry pond.

The absolute disintegration in real process is provided rather difficultly. The disintegration process is poorly controllable in volume. If to supervise the submission of capacity (even with account of efficiency), the part of a material will achieve a stage of absolute disintegration, the size of other parts can remain considerably exceeded of required fineness. At non-uniform distribution of useful component (the particles of gold in goldbearing sand of placer deposit) are probable over optimism high losses. For similar conditions of gold-bearing sand dressing the achievement of a repeatedly exceeding degree of disintegration is more acceptable.

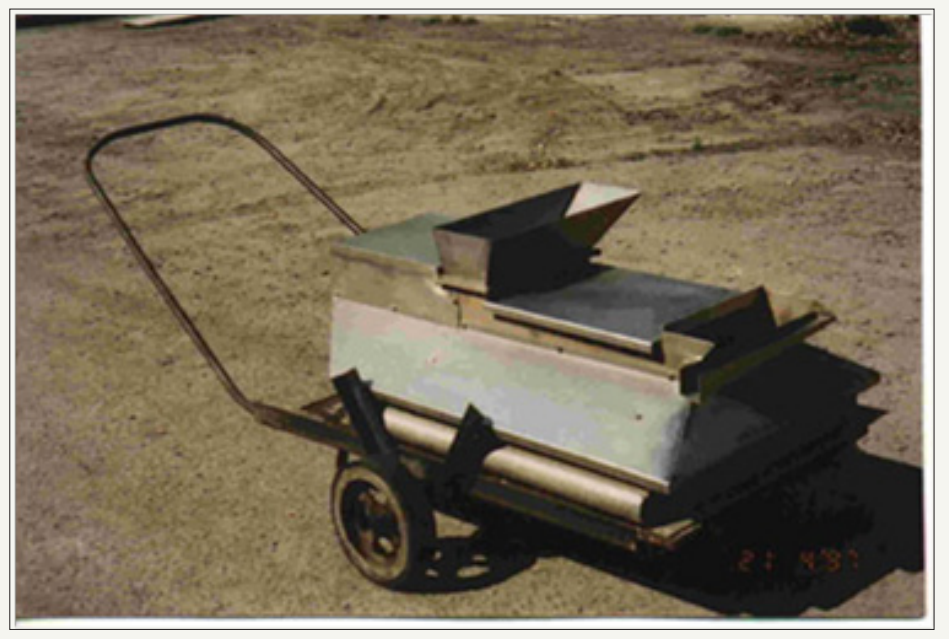

Figure 3: Laboratory shock-acoustic installation. 
The size was determined mean by really using disintegration method. The analysis of disintegration methods shows advantages of acoustic processing of clay. The principle of acoustic influence allows achieving high meanings of oscillatory speed and acceleration in the large technological volumes in high sound pressure. At the passage of acoustic fluctuations the spatial skeleton of a process able material collapses from the high speed of deformation, which take a place owing to break of pressure and movement speed of particles to jump. The distinctive feature of an acoustic method of disintegration is at the capacity of a wave exceeding critical meaning, in a medium where appear the shock waves of local action are formed which causing highly destroying influences on objects, contiguous to this zone. In acoustic methods the low-frequency range is more acceptable. First of all, the basic advantage acoustic treatment in a low-frequency range is a low factor of attenuation of waves [7], it allows to process a material in the large technological volumes. Because of the thyrotrophic properties of clay, a single disruption of the bonds between the particles is not enough. In the space between the particles in the case of broken bonds, free water must be introduced. Only in this case the connection will be closed to a new free level. Due to the fact that clays have thyrotrophic properties, then a single disruption of the bonds between the particles is not enough. All connections are restored. In the space between the particles in the case of broken bonds, free water must be introduced. Only in this case the connections of the particles will be closed at a new free level. Only acoustic processing is not enough. The delivery of water to the clay particles in aggregates can be carried out by vibration or mixing. The researches processing of a clay material in a way of acoustic influence in laboratory installation (Figure 3) is shown a basic opportunity of application of such method in scales accepted to use in industrial conditions.

Laboratory installation generates low-frequency fluctuations in the range of $23-28 \mathrm{~Hz}$ by clasping an elastically compressed plate in the working chamber. This version of the generation of acoustic pulses is accompanied by vibration mixing. Processing can be carried out in flow mode. The results of laboratory studies have shown that the use of this method provides complete disinfection of pure clay material in 3-5 times lower costs with minimal water consumption per process (in limit 1:1).

\section{Conclusion}

In accounts of potential energy of interaction of disperse particles the only basic molecular Van der Waals' attractive forces are taken into account (without electro superficial equilibrium forces of pushing away). With account of the natural size of particles of clay material the absolute disintegration requires application of energy influence not less than $300 \mathrm{~kJ} / \mathrm{m} 3$. This meaning of necessary energy does not take into account such properties of a researched material as thixotropy and geological features of a place of natural material localization and mineral composition. Nevertheless, the order of size of energy allows predicting power parameters of the ore dressing process. Among existing disintegration methods and means of a clay material a method of acoustic processing favorably differs. On the experimental setup, the actual specific energy costs were higher than the theoretical ones by 2 to 3.5 times. During experimental researches on laboratory installation was established, that most difficultly collapse. This clay type has very thin particles. The efficiency of disintegration of hidromica clay grows at $15 \%$, and of montmorillonite clay at $30 \%$ in comparison with kaolin clay.

\section{References}

1. Myazin VP (1995) Increase of efficiency of processing of clay goldbearing sands. Chita, Russia, p. 108.

2. Troickiy VV (1978) Washing of minerals. Nedra, Moscow, Russia, p. 225.

3. Arene VZ (1975) Mine technical methods of production of minerals. Nedra, Moscow, Russia, p. 235.

4. Worell W (1978) Clay and ceramic raw material. Mir, Moscow, Russia, p. 237.

5. Shorohov SM (1973) Technology and complex mechanization of exploitation of placer deposits. Nedra, Moscow, Russia, p. 768.

6. Sulin GA (1974) Technics and technological of exploitation of placer deposits by open-pit method. Nedra, Moscow, Russia, p. 231.

7. Shulgin AI, Nazarova LI, Rehtman VI (1987) Acoustic technology in dressing of minerals. Nedra, Moscow, Russia, p. 232.

8. Shvarcev SL (1996) Common hydrogeology. Nedra, Moscow, Russia, p.423.

9. Pyzh VA (1985) About shock-waves in bentonite suspension. Colloidal magazine, Russia, pp. 894-900.
Creative Commons Attribution 4.0 International License

For possible submissions Click Here

\section{Submit Article}

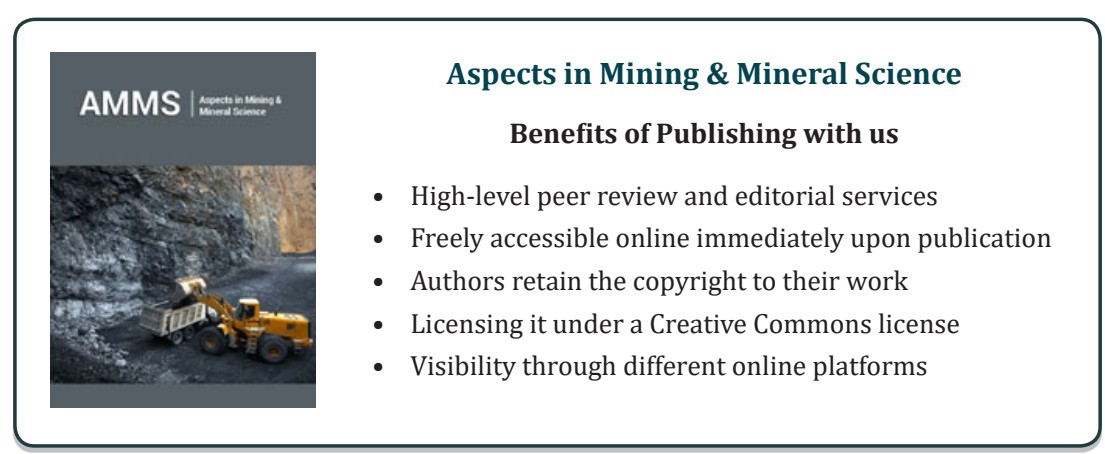

